\title{
TOXICIDAD DE NUEVE PLANTAS Y DE LA CIPERMETRINA EN GORGOJOS DE PRODUCTOS ALMACENADOS Y EN LA PULGA DEL AGUA DAPHNIA MAGNA EN EL PERÚ
}

\author{
Iannacone José \\ Lorena Alvariño, \\ Hildebrando Ayala \\ Neil Salazar
}

\begin{abstract}
RESUMEN
Se realizaron bioensayos para evaluar el efecto tóxico de diferentes sustancias químicas sobre Sitophilus zeamais Motschulsky, 1855, S. oryzae Schoenherr, 1838 (Coleoptera: Curculionidae), Stegobium paniceum (Linneus, 1761) (Coleoptera: Anobiidae) y Daphnia magna Straus, 1820 (Cladocera: Daphnidae). Los ensayos biológicos incluyeron evaluaciones del: 1) efecto insecticida de tres plantas: zapallo (Cucurbita maxima Duch. Ex Lam, Cucurbitaceae), eucalipto (Eucalyptus globulus Labill, Myrtaceae) y coca (Erythroxylon coca [Lamark], Erythroxylaceae) sobre adultos de S. zeamais y de S. paniceum, en bioensayos de mortandad bajo condiciones de laboratorio hasta $120 \mathrm{~h}$ de exposición. Se evaluaron estas tres plantas bajo las cinco formulaciones siguientes: polvo seco (1,6 g·10 g de maíz), extractos acuosos en frío (20\% p/v), infusión (20 \% p/v), cocción $(20 \% \mathrm{p} / \mathrm{v})$ y extracto etanólico $(0,2 \mathrm{~g} \cdot 1 \mathrm{~mL}$ de etanol en $10 \mathrm{~mL}$ de agua). A las concentraciones seleccionadas ninguna de las tres plantas presentó efecto sobre S. zeamais y S. paniceum. 2) efecto insecticida, repelente y atrayente de seis plantas: hoja y flor de floripondio (Brugmansia candida Pers., Solanaceae), hoja y flor de ruda (Ruta graveolens L., Rutaceae), semilla de higuerilla (Ricinus communis L., Euphorbiaceae), bulbo de ajo (Allium sativum L, Liliaceae), hoja de muña (Minthostachys setosa (Briquet) Epling, Lamiaceae) y hojas de eucalipto sobre adultos de $S$. zeamais, bajo condiciones de laboratorio entre $1 \mathrm{~h}$ a $120 \mathrm{~h}$ de exposición para mortandad y 1 h de exposición para repelencia-atracción. Ninguno de los extractos acuosos en infusión mostró efectos estadísticamente significativos de mortandad en comparación con el control. El extracto de eucalipto y de hoja de floripondio produjeron repelencia sobre de los gorgojos. El resto de extractos mostraron efectos atrayentes. 3) efecto tóxico de la cipermetrina al 1,3\% sobre $S$. zeamais y $S$. oryzae, mostrando a $24 \mathrm{~h}$ valores cercanos al $40 \%$ de mortalidad en comparación con el control.4) la actividad biológica de toxicidad aguda a 48 $\mathrm{h}$ de exposición empleando eucalipto, coca, zapallo, muña y granado (Punica granatum L. Punicaceae) en neonatos de la pulga de agua $D$. magna. Se observó la mayor actividad en la formulación acuosa de muña sobre D. magna.
\end{abstract}

Palabras claves: Daphnia, extractos vegetales, insecticidas botánicos, Sitophilus, Stegobium.

\section{SUMMARY}

Bioassays were performed to evaluate toxic effect of different chemical substances on Sitophilus zeamais Motschulsky, 1855, S. oryzae Schoenherr, 1838 (Coleoptera: Curculionidae), Stegobium paniceum (Linneus, 1761) (Coleoptera: Anobiidae) and Daphnia magna Straus, 1820 (Cladocera: Daphnidae). Biological assays included the next evaluations: 1) insecticide effect of three plants: great pumpkin (Cucurbita maxima Duch. Ex Lam, Cucurbitaceae), eucalyptus (Eucalyptus globulus Labill, Myrtaceae) and coca (Erythroxylon coca [Lamark], Erythroxylaceae) on adults of S. zeamais and S. paniceum, on mortality bioassays at laboratory conditions until $120 \mathrm{~h}$ of exposure. Three plants were evaluated on the next five formulations: dry dust (1.6 $\mathrm{g} \cdot 10$

\footnotetext{
${ }^{1}$ Laboratorio de Invertebrados. Facultad de Ciencias Biológicas. Universidad Ricardo Palma.

Av. Benavides 5440, Santiago de Surco, Lima, Perú. E-mail: joseiannacone@gmail.com
} 
g of corn), watery extracts on cold (20\% w/v), infusion (20\% w/v), baking (20\% w/v) and ethanolic extract $(0.2 \mathrm{~g} \cdot 1 \mathrm{~mL}$ of ethanol in $10 \mathrm{~mL}$ of water). At selected concentrations none of three plants presented effect on S. zeamais and S. paniceum. 2) insecticide, repellence and attraction of six plants: leaves and flower of angel's Trumpet (Brugmansia candida Pers., Solanaceae), leaves and flower of rue (Ruta graveolens L., Rutaceae), seeds of perennial castor (Ricinus communis L., Euphorbiaceae), bulb of Garlic (Allium sativum L, Liliaceae), leave of muña (Minthostachys setosa (Briquet) Epling, Lamiaceae) and leave of eucalypt on adults of $S$. zeamais, on laboratory conditions between $1 \mathrm{~h}$ to $120 \mathrm{~h}$ exposure of mortality and $1 \mathrm{~h}$ exposure for repellenceattraction. None of watery extracts of infusion showed significantly statistical mortality effects in comparison to control. Eucalyptus and angel's trumpet leaves extract produced repellence on weevils. The rest of extracts showed effects of attraction. 3) Toxic effect of cypermethrine at $1.3 \%$ on S. zeamais and S. oryzae, showed at $24 \mathrm{~h}$ values near to $40 \%$ of mortality in comparison to control. 4) biological activity of acute toxicity at $48 \mathrm{~h}$ of exposure employing eucalyptus, coca, great pumpkin, muña and Pomegranate (Punica granatum L. Punicaceae) on neonates of water flea D. magna. More activity was observed on watery formulation of muña on D. magna.

Key words: Daphnia, botanical extracts, botanical insecticide, Sitophilus, Stegobium.

\section{INTRODUCCIÓN}

Uno de los principales problemas de los agricultores en los países del tercer mundo es la destrucción de las cosechas de los granos almacenados en calidad y cantidad por roedores, insectos, hongos y bacterias entre un 20\% a 80\% (Silva et al., 2003, 2006; Ngamo et al., 2007). Una de las plagas de mayor relevancia en granos almacenados es Sitophilus zeamais Motschulsky (Coleoptera: Curculionidae) (Salvadores et al., 2007). Esta especie produce perdida de peso en el grano, reducción en el valor estético y en el mercado, disminución en la germinación y en el valor nutritivo del maíz (Udo, 2005; Akob \& Ewete, 2007). Otras plagas de importancia en granos almacenados son Stegobium paniceum (Linnaeus, 1758) (Gunasekaran \& Rajendran, 2005; Gamalie, 2007) y Sitophilus oryzae (Mazzuferi, 2000; Ngamo et al., 2007; Salem et al., 2007).

Es necesario buscar métodos alternativos armónicos con la realidad de cada país, que sean baratos y altamente disponibles (Silva et al., 2004, 2006; Akob \& Ewete, 2007). Muchos insecticidas sintéticos son teratogénicos, mutagénicos, carcinogénicos y afectan la salud de las personas que los emplean (Asawalam, 2006; Iloba \& Ekrakene, 2006; Asawalam et al., 2007). Por ende es vital buscar alternativas que sean accesibles y de bajo impacto para el control de granos almacenados (Ngamo et al., 2007). Una alternativa es emplear plantas biocidas que presenten compuestos químicos secundarios y activos contra las plagas (Awoyinka et al., 2006; Rahman et al., 2007), muchas de las cuales no han sido adecuadamente evaluadas y es importante su revalorización como fuente de sustancias con propiedades insecticidas, repelentes, deterrentes de la oviposición y alimentación, y reguladores de crecimiento (Silva et al., 2005; Asawalam, 2006). Las plantas biocidas son una alternativa armónica con el desarrollo sostenible (Salvadores et al., 2007). Daphnia magna Strauss, 1820 (Crustácea: Daphniidae) es una especie de cladócero partenogenética usada ampliamente en ensayos de ecotoxicidad (Tonkopii \& Iofina, 2007), para evaluar sustancias químicas puras, aguas residuales domésticas e industriales, aguas superficiales o subterráneas, agua potable, y lixiviados entre otros (Iannacone \& Alvariño, 2007a; Iannacone et al., 2007). Su uso es debido a varias razones: 1 ) amplia distribución geográfica, 2) importante papel en la comunidad zooplanctónica, 3) facilidad de cultivo, 4) corto ciclo de vida con la producción de un alto número de crías (Castillo, 2004).

El objetivo de este trabajo fue evaluar bajo condiciones de laboratorio, 1) el efecto toxicológico de tres plantas: zapallo (Cucurbita maxima Duch. Ex Lam, Cucurbitaceae), eucalipto (Eucalyptus globulus Labill, Myrtaceae) y coca (Erythroxylon coca [Lamark], Erythroxylaceae) sobre adultos de $S$. zeamais y de $S$. paniceum, en bioensayos de mortandad bajo condiciones de laboratorio hasta 120 h de exposición. 2) efecto insecticida, repelente y atrayente de seis plantas: hoja y flor de floripondio (Brugmansia candida Pers., Solanaceae), hoja y flor de ruda (Ruta graveolens L., Rutaceae), semilla de higuerilla (Ricinus communis L., Euphorbiaceae), bulbo de ajo (Allium sativum L, Liliaceae), hoja de muña (Minthostachys setosa (Briquet) Epling, Lamiaceae) y hojas de eucalipto sobre adultos de $S$. zeamais, 3) efecto tóxico de la cipermetrina al 1,3\% sobre $S$. zeamais y $S$. oryzae, mostrando a $24 \mathrm{~h}$ valores cercanos al $40 \%$ de mortalidad en comparación con el control, y 4) la actividad biológica de toxicidad aguda a $48 \mathrm{~h}$ de exposición empleando 
eucalipto, coca, zapallo, muña y granado (Punica granatum L. Punicaceae) en neonatos de la pulga de agua $D$. magna.

\section{MATERIAL Y MÉTODOS}

Los bioensayos se realizaron en el Laboratorio de Invertebrados de la Facultad de Ciencias Biológicas de la Universidad Ricardo Palma entre agosto del 2004 a febrero del 2008.

Gorgojos de granos almacenados. Las crianzas de $S$. zeamais, $S$. oryzae y de $S$. paniceum fueron iniciadas a partir de adultos procedentes del Mercado Jorge Chávez, La Victoria, Lima, Perú, y alimentadas con maíz variedad amarillo duro para pollo y variedad cancha. Posteriormente los individuos fueron trasladados al laboratorio, separados por especie y colocados en envases plásticos de $1 \mathrm{~L}$ de capacidad, y mantenidos a una temperatura aproximada de $25^{\circ} \mathrm{C}$ $\pm 3^{\circ} \mathrm{C}$, para favorecer la reproducción, oviposición y obtención de los adultos requeridos para realizar los bioensayos toxicológicos (Iannacone et al., 2005).

Pulga del agua. Hembras adultas de esta especie se obtuvieron del acuario «Cleo» procedente del distrito de Lince, Lima, Perú, y se llevaron al laboratorio en recipientes plásticos de $2 \mathrm{~L}$ de capacidad. Hembras partenogenéticas se colocaron en el medio nutritivo ADaM. La preparación del medio se realizó de la siguiente forma: 9,9 g de sales obtenidas por evaporación del agua de mar se adicionaron a $60 \mathrm{~L}$ de agua de grifo declorinada, reposada y hiperoxigenada durante $24 \mathrm{~h}$. Luego se agregó $138 \mathrm{~mL}$ de una solución de cloruro de calcio $\left(117,6 \mathrm{~g} \cdot \mathrm{L}^{-1}\right), 132 \mathrm{~mL}$ de una solución de bicarbonato de sodio $\left(25,2 \mathrm{~g} \cdot \mathrm{L}^{-1}\right)$ y $6 \mathrm{~mL}$ de una solución de Oxido de Selenio $\left(0,07 \mathrm{~g} \mathrm{~L}^{-1}\right)$ (Klüttgen et al., 1994). Plantas. Las plantas utilizadas en los ensayos toxicológicos provinieron de distintos mercados y Parques de Lima, Perú (Tabla 1). 250 g de bulbo, hojas, flor o semilla de cada especie según lo indicado en la Tabla 1 para cada uno de los diferentes ensayos, fueron secadas en estufa a $37^{\circ} \mathrm{C}$, por $70 \mathrm{~h}$ aproximadamente, hasta obtener un peso constante por la pérdida de agua. Posteriormente las hojas fueron trituradas en un mortero (Haldenwanger ${ }^{\circledR}$ ) y el residuo obtenido fue pasado secuencialmente por 2 tamices de $500 \mu$ y $250 \mu$. Las muestras fueron envasadas en frascos de vidrio color ámbar para evitar la fotolisis, rotuladas, y guardadas a temperatura de $25^{\circ} \mathrm{C} \pm 3^{\circ} \mathrm{C}$ hasta el día a ser utilizadas en los bioensayos (Iannacone \& Lamas, 2003).

\section{BIOENSAYOS}

1) Efecto insecticida de tres plantas (Cucurbita maxima, Eucalyptus globulus y Erythroxylon coca) sobre adultos de $S$. zeamais y de $S$. paniceum.

En seco: Se colocaron 10 individuos de cada especie de gorgojo en envases cuadrangulares de plástico con cuatro réplicas. La distribución del polvo seco de las plantas se realizó de la siguiente manera: 0,1 g, 0,2 g, 0,4 g, 0,8 g y 1,6 g por cada 10 g de maíz en cada una de las cuatro replicas, y cinco dosis más un control (Iannacone et al., 2004, 2006); los insectos fueron controlados cada 24 h durante 5 días ( $=120$ h), según la técnica sugerida por Mazzonetto \& Vendramim (2003).

En acuoso: El extracto botánico acuoso crudo, fue preparado con agua destilada $(\mathrm{pH} 6 \pm 0,5)$ a cinco concentraciones, y filtrados a través de un papel fino (Whatman ${ }^{\circledR} \mathrm{N}^{\circ} 5$, USA). Solo se usaron extractos acuosos que habían sido recientemente preparados (no más de 48 h), debido a que microorganismos fúngicos pudieran afectar la calidad de los mismos (Iannacone \& Lamas, 2003). Se colocaron $10 \mathrm{~g}$ de maíz previamente remojados por 5 seg por cada concentración y cada tratamiento, y se agregaron 10 individuos de cada especie de gorgojo en cada envase con las concentraciones de $1,25 \%$, 2,5 \%, $5 \%$, $10 \%$ y $20 \%$, respectivamente, con cuatro replicas más un control, evaluados cada 24 h durante 5 días (= 120 h), para obtener el efecto de mortalidad. Para los extractos de cocción e infusión en agua destilada se prepararon a las mismas concentraciones que los extractos acuosos a temperatura ambiente, siguiendo las recomendaciones de Iannacone et al. (2004).

Extracto etanólico: Se tomó 0,2 g de cada una de las tres plantas y se colocó en un $1 \mathrm{~mL}$ de etanol y posteriormente en $10 \mathrm{~mL}$ de agua destilada. A partir de los cuales se hicieron las otras cuatro diluciones empleando un factor de 0,5. De igual manera se evaluó cada 24 h durante 5 días (120 h).

2) Efecto insecticida, repelente y atrayente de seis plantas (Brugmansia candida, Ruta graveolens, Ricinus communis, Allium sativum, Minthostachys setosa y eucalipto) sobre $S$. zeamais. Se usó sobre $S$. zeamais el extracto de infusión acuosos para evaluar la actividad insecticida de seis plantas: B. candida, $R$. graveolens, $R$. communis, A. sativum, $M$. setosa y eucalipto. Se siguió el protocolo y las concentraciones indicadas para extractos acuosos del primer caso. 
Para el ensayo de repelencia y atracción se empleó el método estándar (Asawalam, 2006). Los polvos secos de cada una de las seis plantas fueron disueltos en agua destilada para hacer una solución al 20\% p/ v. Papeles filtros Whatman $\mathrm{N}^{\circ} 40$ de $9 \mathrm{~cm}$ de diámetro fueron cortados en dos mitades. Una de las mitades fue sumergida en el extracto acuoso y la otra mitad en agua destilada. Los discos tratados y no tratados fueron secados a la estufa y colocados en placas de petri, previamente fueron unidas las dos mitades empleando una cinta adhesiva. 10 insectos fueron colocados en el centro de la placa de petri y luego de $1 \mathrm{~h}$ fueron contados los insectos que se encontraron en cada una de las mitades de los discos en l placa de petri. El porcentaje de repelencia fue calculado usando la siguiente fórmula de Abbotts:

Porcentaje de repelencia $=[(\mathrm{A}-\mathrm{B}) / \mathrm{A}] \mathrm{x} 100$

A = Promedio del número de insectos presentes en la porción no tratada. $\mathrm{B}=$ Promedio del número de insectos presentes en la porción no tratada.

El porcentaje de repelencia fue categorizado de acuerdo a la siguiente escala:

\begin{tabular}{llll}
\hline Clase & Repelencia & Clase & Repelencia \\
\hline Porcentaje & $(\%)$ & Porcentaje & $(\%)$ \\
0 & $>0,01$ a 0,10 & III & 40,1 a 60 \\
I & 0,10 a 20 & IV & 60,1 a 80 \\
II & 20 a 40 & V & 80,1 a 100 \\
\hline
\end{tabular}

En el caso de dar resultados negativos se consideró como atracción.

3) Efecto tóxico de la cipermetrina al 1,3\% sobre $S$. zeamais y $S$. oryzae.

Se usó la cipermetrina en formulación tipo fumígena. La composición química es 1,3\% de cipermetrina y 98,7\% de inertes (Iannacone \& Alvariño, 2007b). Se empleó una pastilla por cada por habitación de 9 m², la cual fue colocada sobre el suelo en un trozo de mayólica de $20 \mathrm{~cm}$ x $20 \mathrm{~cm}$. Los ensayos con ambas especies de gorgojos $S$. zeamais y $S$. oryzae fueron realizados en simultáneo. El control consistió en el empleo de una habitación de $9 \mathrm{~m}^{2}$ sin la aplicación de la cipermetrina. En cada recipiente cuadrangular de $40 \mathrm{~mL}$ fueron colocados 10 gorgojos. Se realizaron 25 orificios en cada uno de los cuatro lados laterales y en la tapa de cubierta. 13 recipientes fueron colocados sobre el suelo a $50 \mathrm{~cm}$ de separación entre recipientes. Las lecturas se realizaron a las $3 \mathrm{~h}, 6 \mathrm{~h}$ y 24 h de exposición a la cipermetrina fumígena.

4) Actividad biológica de toxicidad aguda a $48 \mathrm{~h}$ de exposición empleando eucalipto, coca, zapallo, muña y granado (Punica granatum $\mathrm{L}$. Punicaceae) en neonatos de la pulga de agua $D$. magna.

La duración total de la prueba fue de 48 h de exposición para todos los casos. A cada envase circular de $250 \mathrm{~mL}$ se procedió a agregar $100 \mathrm{~mL}$ de cada una de las concentraciones de las sustancias químicas empleadas, a los que se transfirieron diez neonatos de $D$. magna. Se usó como criterio de mortalidad la carencia de movilidad o la ausencia de ritmo cardiaco a $15 \mathrm{~s}$ de observación al microscopio estereoscopio (Castillo, 2004).
Diseño experimental y tratamiento estadístico. Las pruebas de toxicidad aguda se evaluaron en concentraciones nominales para cada planta a través de un ANDEVA, con el modelo aditivo lineal, empleando un diseño en bloque completamente aleatorizado (DBCR) de 6 concentraciones-dosis $\mathrm{x}$ 4 repeticiones. Los datos fueron transformados a arcoseno (porcentaje de mortalidad de adultos/100) ${ }^{0,5}$ antes del análisis, para estabilizar el error de la varianza. En el caso de existir diferencias significativas entre las repeticiones y entre los tratamientos se hicieron pruebas de diferencias verdaderamente significativas (DVS) de Tukey. Solo cuando en los bioensayos se encontraron mortalidades diferentes de cero en el control los análisis estadísticos se realizaron con los valores ajustados según la fórmula de Abbott (Iannacone \& Lamas, 2003). Se empleó el paquete estadístico SPSS en español, versión 15,0 para el cálculo de la estadística descriptiva e inferencial.

\section{RESULTADOS}

Se encontró que C. máxima, E. globulus y E. coca en polvo seco, en extracto acuoso, extracto en infusión, en cocción, y etanólico no produjeron efectos sobre la mortalidad de $S$. zeamais y $S$. paniceum a 120 h de exposición (Tabla 2).

Ninguna de las seis plantas ensayadas en extracto de infusión produjo efecto en la mortalidad de $S$. zeamais a 120 h de exposición (Tabla 3). Las hojas de $B$. candida y E. globulus ocasionaron repelencia 
en escala II y III, respectivamente sobre $S$. zeamais. La flor de $B$. candida, la hoja y flor de $R$. graveolens, las semillas de $R$. communis, el bulbo de $A$. sativum y la hoja de $M$. setosa produjeron contrariamente un efecto atrayente (Tabla 3).

A 3 h, 6 h y 24 h se observó igual mortalidad por la cipermetrina en adultos de $S$. zeamais que en $S$. oryzae. Para ambos gorgojos se observaron diferencias en los porcentajes de mortalidad entre los controles y los tres periodos de exposición ensayados (Tabla 4).

Con relación a los extractos de infusión de las cinco plantas evaluadas a las 24 h de exposición se vio un $80 \%$ de mortalidad sobre $D$. magna para las hojas de E. coca y para las flores de P. granatum; así como un $90 \%$ de mortalidad para $M$. setosa. A las $48 \mathrm{~h}$, todas las plantas ocasionaron un 100\% de mortalidad en D. magna (Tabla 5).

\section{DISCUSIÓN}

La literatura científica señala diversas plantas promisorias con propiedades biocidas sobre gorgojos de granos almacenados, principalmente en $S$. zeamais (Oliveira et al., 2003; Akon \& Ewete, 2007). Las hojas de E. globulus ocasionaron repelencia en escala III sobre S. zeamais. Se ha encontrado ausencia de efecto en la mortalidad de $S$. zeamais por acción de polvos secos, extractos acuosos, de infusión y cocción de E. globulus a 120 h de exposición (Iannacone et al., 2006). Lee et al. (2004) evaluó los efecto fumigantes de Eucalyptus blakelyi sobre huevos, larvas, pupas y adultos de $S$. oryzae. El efecto tóxico de E. blakeli sobre $S$. oryzae es atribuido al cineol. Sin embargo, los análisis cromatográficos han detectado más de 57 sustancias en E. blakeli. En Sitophilus granarius (Linnaeus) ha sido evaluado el efecto en la emergencia de los adultos, daño en las semillas, perdida de peso e inhibición por acción de Eucalyptus macrorhyncha (Rahman et al., 2003). De igual manera, se ha encontrado mortalidad significativa en los adultos de S. zeamais bajo tratamientos con Eucalyptus sargentii, Eucalyptus intertexta, Eucalyptus camaldulensis, Eucalyptus grandis y Eucalyptus saligna (Akob \& Ewete, 2007; Negaban \& Moharramipour, 2007). De igual forma las hojas de B. candida ocasionaron repelencia en escala II sobre S. zeamais. PIER (2008) realizó una evaluación de riesgo toxicológica de $B$. candida. Se ha observado mortalidad y repelencia de larvas del gorgojo Rhynchophorus palmarum por acción de $B$. candida (Pérez \& Iannacone, 2006). Pérez \& Iannacone (2008) encontraron bajo efecto en la mortalidad, pero alto efecto en la repelencia sobre las larvas del lepidóptero Eupalamides cyparissias a 24 h de exposición.

Con relación a los extractos de infusión de las cinco plantas evaluadas a las 24 h de exposición sobre $D$. magna se vio un $90 \%$ de mortalidad para $M$. setosa. De igual manera, Sutil et al. (2006) encontraron toxicidad de los aceites esenciales de Minthostachys verticillata sobre Artemia salina y en líneas celulares Vero y HEp-2. De igual manera, Ciccia et al. (2000) evaluó la toxicidad de Minthostachys setosa sobre Aedes aegypti. Alkire et al. (1994) y Schmidt-Lebuhn (2008) evaluaron el efecto toxicologico y la composición bioquímica del género Minthostachys.

\section{LITERATURA CITADA}

AKOB, C. A. \& EWETE, F.K. 2007. The efficacy of ashes of tour locally used plant materials against Sitophilus zeamais (Coleoptera: Curculionidae) in Cameroon. International Journal of Tropical Insect Science. 27: 21-26. ALKIRE, B.H.; TUCKER, A.O. \& MACIARELLO, M.J. 1994. Tipo, Minthostachys mollis (Lamiaceae): an Ecuadorian mint. Economic Botany. 48: 60-64.

ASAWALAM, E.F. 2006. Insecticidal and repellent properties of Piper guineense seed oils extract for the control of maize weevil, Sitophilus zeamais. Electronic Journal of Environmental, Agricultural and Food Chemistry. 5: 1389-1394.

ASAWALAM, E.F.; EMOSAIRUE, S.O.; EKELEME, F. \& WOKOCHA, R.C. 2007. Insecticidal effects of powdered parts of eight Nigerian plant species against maize weevil Sitophilus zeamais Motschulsky (Coleoptera: Curculionidae). Electronic Journal of Environmental, Agricultural and Food Chemistry. 6: 2526-2533.

AWOYINKA, O.A.; OYEWOLE, I.O.; AMOS, B.M.W. \& ONASOGA, O.F. 2006. Comparative pesticidal activity of dichloromethane extracts of Piper nigrum against Sitophilus zeamais and Callosobruchus maculatus. African Journal of Biotechnology. 5: 2446-2449.

CASTILLO, G. 2004. Ensayos toxicológicos y métodos de evaluación de calidad de aguas. Estandarización, intercalibración, resultados y aplicaciones. IMTA. México.

CICCIA, G.; COUSSIO, J. \& E. MONGELLI. 2000. Insecticidal activity against Aedes aegypti larvae of some medicinal South American plants. Journal of Ethnopharmacology. 72: 185-189.

GAMALIE, G. 2007. Monitoring of Stegobium paniceum Linné 1758 (Coleoptera: Anobiidae) 
on book deposits by means of traps. Analele ${ }^{a}$ tiinpifice ale Universitãbii «Al. I. Cuza»Ia ${ }^{\circ}$. 53: 89-96.

IANNACONE, J. \& LAMAS, G. 2003. Efectos toxicológicos de extractos de molle (Schinus molle) y lantana (Lantana camara) sobre Chrysoperla externa (Neuroptera: Chrysopidae), Trichogramma pintoi (Hymenoptera: Trichogrammatidae) y Copidosoma koehleri (Hymenoptera: Encyrtidae), en el Perú. Agricultura Técnica (Chile). 63: 347-360.

IANNACONE, J.; AYALA, H.; ALVAREZ, J.; LEYVA, O. \& BAJALQUE, E. 2004. Cuatro plantas biocidas sobre Sitophilus zeamais Motschulsky, 1855 (Coleoptera: Curculionidae) y Stegobium paniceum (Linnaeus, 1761) (Coleoptera: Anobiidae), en el Perú. Wiñay Yachay (Perú). 8: 16-27.

IANNACONE, J.; AYALA, H. \& ROMÁN, A. 2005. Efectos toxicologicos de cuatro plantas sobre el gorgojo del maíz Sitophilus zeamais Motschulsky 1855 (Coleoptera: Curculionidae) y sobre el gorgojo de las galletas Stegobium paniceum (Linnaeus 1761) (Coleoptera: Anobiidae) en Peru. Gayana. 69: 234-240.

IANNACONE, J.; AYALA, H.; ROMÁN, A.; CARRILLO, R.; SOTO, J.C.; SALCEDO, C.; ESCALANTE, C.; VALLEJOS, M. \& PÉREZ, D. 2006. Efecto insecticida de cinco plantas sobre el gorgojo del maíz Sitophilus zeamais Motschulsky, 1855 (Coleoptera: Curculionidae) en Perú. Scientia (Lima). 8: 197-206.

IANNACONE, J. \& ALVARIÑO, L. 2007a. Ecotoxicidad acuática de dos colorantes y de tres antiparasitarios de importancia en acuicultura en Daphnia magna. Ecología Aplicada. 6: 101-110.

IANNACONE, J. \& ALVARIÑO, L. 2007b. Integración del control químico y etológico para la supresión poblacional de Blatella germanica (Linnaeus) (Dictyoptera: Blatellidae) en Lima, Perú. Parasitología Latinoamericana. 62: 7-15.

IANNACONE, J.; ALVARIÑO, L.; SOTO, J.C. \& SALCEDO, C. 2007. Efecto toxicológico del «Sachayoco», Paullinia clavigera (Sapindaceae) sobre Daphnia magna y sobre dos controladores biológicos de plagas agrícolas. Journal of Brazilian Society of Ecotoxicology. 2: 15-25.

ILOBA, B.N. \& EKRAKENE, T. 2006. Comparative assessment of insecticidal effect of Azadirachtina indica, Hyptis suaveolens and Ocimum gratissimum on Sitophilus zeamais and Callosobruchus maculatus. Journal of Biological Sciences. 6: 626-630.
KLÜTTGEN, B.; DÜLMER, U.; ENGELS, M. \& RATTE, H.T. 1994. ADaM, an artificial freshwater for culture of zooplankton. Water Research. 28: 743-746.

LEE, B.H.; ANNIS, P.C.; TUMAALII, F.A. \& LEE, S.E. 2004. Fumigant toxicity of Eucalyptus blakelyi and Melaleuca fulgens essential oils and 1,8-cineole against different development stages of the rice weevils Sitophilus oryzae. Phytoparasitica. 32: 498-506.

MAZZONETTO, F. \& VENDRAMIM, J.D. 2003. Efeito de pós de origem vegetal sobre Acanthoscelides obtectus (Say) (Coleoptera: Bruchidae) em feijão armazenado. Neotropical Entomology. 32: 145-149.

MAZZUFERI, V.; CARRERAS, J. \& CASANOVES, F. 2000. Uso de fosfamina para el control de Sitophilus oryzae (L.) (Coleoptera: Curculionidae) en semillas de garbanzo (Cicer arietinum L.) y efectos sobre su viabilidad. Agriscientia. 17: 65-68.

NEGAHBAN, M. \& MOHARRAMIPOUR, S. 2007. Fumigant toxicity of Eucalyptus intertexta, Eucalyptus sargentii and Eucalyptus camaldulensis against stored-product beetles. Journal of Applied entomology. 131: 256-261.

NGAMO, T.S.L.; NGATANKO, I.; NGASSOUM, M.B.; MAPONGMESTSEM, P.M. \& HANCE, T. 2007. Persistence of insecticidal activities of crude essential oils of three aromatic plants toward four major stored product insect pests. African Journal of Agriculture Research. 2: 173-177.

OLIVEIRA, S.; VENDRAMIM, J.D.; RIBEIRO, J.I. \& DOS SANTOS, J.B. 2003. Bioatividade de diversos pós de origem vegetal em relação a Sitophilus zeamais Mots. (Coleoptera: Curculionidae). Ciên Agr. 27: 1231-1236.

PÉREZ, D. \& IANNACONE, J. 2006. Efectividad de extractos botánicos de diez plantas sobre la mortalidad y repelencia de larvas de Rhynchophorus palmarum L., insecto plaga del pijuayo Bactris gasipaes Kunth en la amazonía del Perú. Agricultura. Técnica (Chile). 66: 21-31.

PÉREZ, D. \& IANNACONE, J. 2008. Mortalidad y repelencia en Eupalamides cyparissias (Lepidoptera: Castniidae), plaga de la palma aceitera Elaeis guineensis, por efecto de diez extractos botánicos. Revista de la Sociedad Entomológica Argentina. 67: 41-48

PIER (Pacific Island Ecosystems at Risk). 2008. Brugmansia x candida. Risk Assessment Results. En: http://www.hear.org/pier/wra/ pacific/brugmansia_candida_htmlwra.htm leído el 10 de octubre del 2008. 
RAHMAN, M.A.; TALEB, M.A. \& BISWAS, M.M. 2003. Evaluation of botanical products as grain protectant against grain weevil, Sitophilus granarius (L.) on wheat. Asian Journal of Plant Sciences. 2: 501-504.

RAHMAN, S.S.; RAHMAN, M.M.; BEGUM, S.A.; KHAN, M.M.R. \& BHUIYAN, M. H. 2007. Investigation of Sapindus mukorossi extracts for repellency, insecticidal activity and plant growth regulatory effect. Journal of Applied Sciences Research. 3: 95-101.

SALVADORES, U.Y.; SILVA, A.G; TAPIA, V.M. \& HEPP, G.R. 2007. Polvos de especies aromáticas para el control del gorgojo del maíz Sitophilus zeamais Motschulsky, en trigo almacenado. Agricultura Técnica (Chile). 67: 147-154.

SALEM, S.A.; ABOU-ELA, R.G.; MATTER, M.M. \& EL-KHOLY, M.Y. 2007. Entomocidal effect of Brassica napus extracts on two store pests, Sitophilus oryzae (L.). and Rhizopertha dominica (Fab.) (Coleoptera). Journal of Applied Sciences Research. 3: 317-322.

SCHMIDT-LEBUHN, A.N. 2008. Ethnobotany, biochemistry and pharmacology of Minthostachys (Lamiaceae). Journal of Ethnopharmacology. 118: 343-353.

SILVA, G.; LAGUNES, A. \& RODRIGUEZ, J. 2003a. Control de Sitophilus zeamais con polvos vegetales solos y en mezclas con carbonato de calcio en maíz almacenado. Ciencia $e$ investigación agraria. 30: 153-160.

SILVA, G.; PIZARRO, D.; CASALS, P. \& BERTI, M. 2003b. Evaluación de plantas medicinales en polvo para el control de Sitophilus zeamais Motschulsky en maíz almacenado. Revista Brasileira de Agrociência. 9: 383-388.

SILVA, G.; GONZALES, G.P.; HEPP, G. R. \& CASALS, B.P. 2004. Control de Sitophilus zeamais Motschulsky con polvos inertes. Agrociencia. 38: 529-536.

SILVA, G.; ORREGO, O.; HEPP, R. \& TAPIA, M. 2005. Búsqueda de plantas com prpiedades insecticidas para el control de Sitophilus zeamais em maíz almacenado. Pesquisa agropecuária brasileira. 40: 11-17.

SILVA, G.; HEPP, G.R.; TAPIA, V.M.; CASALS, B.P.; BUSTOS, F. G. \& OSSES, R.F. 2006. Evaluación de boldo (Peumus boldus Molina) y cal para el control de Sitophilus zeamais Motschulsky. Agrociencia. 40: 219-228.

SUTIL, S.B.; ASTESANO, A.; VOGT, V.; TORRES, C.V.; ZANON, S.M. \& SABINI, L.I. 2006. Minthostachys verticillana: toxicity of its essential oils and major constituents to Artemia salina and cell lines. IECEFYN. 10: 41-42.

TONKOPII, V. \& IOFINA, I. 2008. The usage of Daphnia magna as alternative bioobject in ecotoxicology. Alternatives to Animal Testing and Experimentation.14: 565-567.

UDO, I.O. 2005. Evaluation of the potential of some local spices as stored grain protectants against the maize weevil Sitophilus zeamais Mots (Coleoptera: Curculionidae). Journal of Applied Sciences and Environmental Management. 9: 165-169.

\section{Tabla 1. Especies de plantas evaluadas para el control de gorgojos de granos almacenados y Daphnia magna.}

\begin{tabular}{llllc}
\hline \multicolumn{1}{c}{ Nombre científico } & $\begin{array}{c}\text { Nombre } \\
\text { vernacular }\end{array}$ & \multicolumn{1}{c}{ Familia } & $\begin{array}{c}\text { Parte } \\
\text { empleada }\end{array}$ & $\begin{array}{c}\text { Caso } \\
\text { empleada }\end{array}$ \\
\hline Allium sativum & ajo & Liliaceae & bulbo & 2 \\
Brugmansia candida & floripondio & Solanaceae & Hoja y flor & 2 \\
Cucurbita maxima & zapallo & Cucurbitaceae & semilla & 1 y 4 \\
Erythroxilon coca & coca & Erythroxylaceae & hoja & 1 y 4 \\
Eucalyptus globulus & eucalipto & Myrtaceae & hoja & 1 , 2 y 4 \\
Minthostachys setosa & muña & Lamiaceae & hoja & 2 y 4 \\
Punica granatum & granada & Punicaceae & flor & 3 \\
Ricinus communis & higuerilla & Euphorbiaceae & semillas & 2 \\
Ruta graveolens & ruda & Rutaceae & Hoja y flor & 2 \\
\hline
\end{tabular}

Caso 1 = efecto toxicológico de tres plantas: zapallo (Cucurbita maxima, Cucurbitaceae), eucalipto (Eucalyptus globulus, Myrtaceae) y coca (Erythroxylon coca, Erythroxylaceae) sobre adultos de S. zeamais y de S. paniceum, en bioensayos de mortandad bajo condiciones de laboratorio hasta 120 h de exposición.

Caso 2 = efecto insecticida, repelente y atrayente de seis plantas: hoja y flor de floripondio (Brugmansia candida, Solanaceae), hoja y flor de ruda (Ruta graveolens, Rutaceae), semilla de higuerilla (Ricinus communis, Euphorbiaceae), bulbo de ajo (Allium sativum, Liliaceae), hoja de muña (Minthostachys setosa, Lamiaceae) y hojas de eucalipto sobre adultos de $S$. zeamais,

Caso 4 = actividad biológica de toxicidad aguda a 48 h de exposición empleando eucalipto, coca, zapallo, muña y granado (Punica granatum, Punicaceae) en neonatos de la pulga de agua D. magna. 
Tabla 2. Porcentaje de mortalidad de las tres plantas evaluadas sobre el gorgojo del maíz Sitophilus zeamais y del gorgojo de la galleta Stegobium paniceum a 120 h de exposición.

\begin{tabular}{lcccccccccc}
\hline \multicolumn{1}{c}{ Tratamientos } & \multicolumn{4}{c}{$\begin{array}{c}\text { Sitophilus zeamais } \\
\text { (\% mortalidad) }\end{array}$} & \multicolumn{4}{c}{$\begin{array}{c}\text { Stegobium paniceum } \\
\text { (\% mortalidad) }\end{array}$} \\
\cline { 2 - 11 } Cucurbitamáxima & A & B & C & D & E & A & B & C & D & E \\
\cline { 2 - 12 } Eucalyptus globulus & 0 & 0 & 0 & 0 & 0 & 0 & 0 & 0 & 0 & 0 \\
Erythroxilon coca & 0 & 0 & 0 & 0 & 0 & 0 & 0 & 0 & 0 & 0 \\
Control & 0 & 0 & 0 & 0 & 0 & 0 & 0 & 0 & 0 & 0 \\
\hline
\end{tabular}

A = Polvo seco (1,6 g. 10 g de maíz). B = Extracto acuoso en frío (20\%). C = Extracto infusión (20\%). D = Extracto cocción (20\%). E = Extracto etanólico (0,2 g $1 \mathrm{~mL}$ de etanol en $10 \mathrm{~mL}$ de agua).

Tabla 3. Porcentaje de mortalidad y repelencia de seis plantas en extracto de infusión evaluadas sobre el gorgojo del maíz Sitophilus zeamais a 120 h de exposición.

\begin{tabular}{lccc}
\hline \multicolumn{1}{c}{ Extracto de infusión (20\%) } & $\begin{array}{c}(\% \\
\text { Mortalidad) }\end{array}$ & $\begin{array}{c}\text { Porcentaje de } \\
\text { repelencia }\end{array}$ & $\begin{array}{c}\text { Escala de } \\
\text { repelencia }\end{array}$ \\
\hline Brugmansia candida (Solanaceae), hoja & 0 & 33,3 & II \\
Brugmansia candida (Solanaceae), flor & 0 & -70 & Atrayente \\
Ruta graveolens(Rutaceae), hoja & 0 & $-35,3$ & Atrayente \\
Ruta graveolens(Rutaceae), flor & 0 & $-66,7$ & Atrayente \\
Ricinus communis (Euphorbiaceae), & 0 & $-22,2$ & Atrayente \\
semillas & & & \\
Allium sativum (Liliaceae), bulbo & 0 & -50 & Atrayente \\
Minthostachys setosa(Lamiaceae), hoja & 0 & $-10,5$ & Atrayente \\
Eucalyptus globulus(Myrtaceae), hoja & 0 & 57,1 & III \\
Control & 0 & 0 & - \\
\hline
\end{tabular}

Valores son signos negativos indican que los extractos fueron atrayentes.

Valores con signos positivos señalan que los extractos fueron repelentes. Escala de repelencia II $=20$ a $40 \%$ de repelencia. Escala de repelencia III = 40 a $60 \%$ de repelencia.

Tabla 4. Toxicidad de la cipermetrina al 1,3\% sobre Sitophilus zeamais y S. oryzae a 3 h, 6 h y 24 h de exposición.

\begin{tabular}{clcc}
\hline $\begin{array}{c}\text { Periodo de } \\
\text { exposición }\end{array}$ & Tratamientos & $\begin{array}{c}\text { S zeamais } \\
\text { (\% mortalidad) }\end{array}$ & $\begin{array}{c}\text { S. oryzae } \\
\text { (\% mortalidad) }\end{array}$ \\
\hline \multirow{2}{*}{$3 \mathrm{~h}$} & Control & $0,76 \mathrm{a}$ & $0 \mathrm{a}$ \\
& cipermetrina & $19,2 \mathrm{bc}$ & $14,6 \mathrm{bc}$ \\
\multirow{2}{*}{$6 \mathrm{~h}$} & Control & $1,98 \mathrm{a}$ & $0 \mathrm{a}$ \\
& cipermetrina & $30 \mathrm{~cd}$ & $26,9 \mathrm{~cd}$ \\
\multirow{2}{*}{$24 \mathrm{~h}$} & Control & $1,98 \mathrm{a}$ & $0 \mathrm{a}$ \\
& cipermetrina & $40,7 \mathrm{~d}$ & $38,5 \mathrm{~d}$ \\
\hline
\end{tabular}

Letras minúsculas iguales en una misma columna indican que los promedios son estadísticamente iguales a un $\mathrm{P}<0,05$ según la prueba de Tukey. 
Tabla 5. Porcentaje de mortalidad de cinco plantas en extracto de infusión evaluadas sobre la pulga del agua Daphnia magna a 120 h de exposición.

\begin{tabular}{lcc}
\hline $\begin{array}{c}\text { Tratamientos a base de extracto infusión } \\
\left(1 \mathrm{~g} \text { del Pde la planta L } \mathrm{L}^{-1}\right.\end{array}$ & $\begin{array}{c}24 \mathrm{~h}(\% \mathrm{de} \\
\text { mortalidad) }\end{array}$ & $\begin{array}{c}48 \mathrm{~h} \text { (\% de } \\
\text { mortalidad) }\end{array}$ \\
\hline Eucalyptus globulus(Myrtaceae), hoja & $85 \mathrm{~b}$ & $100 \mathrm{~b}$ \\
Erythroxilon coca (Erythroxylaceae), hoja & $80 \mathrm{~b}$ & $100 \mathrm{~b}$ \\
Cucurbita maxima (Cucurbitaceae), semilla & $85 \mathrm{~b}$ & $100 \mathrm{~b}$ \\
Minthostachys setosa(Lamiaceae), hoja & $90 \mathrm{~b}$ & $100 \mathrm{~b}$ \\
Punica granatum (Punicaceae), flor & $80 \mathrm{~b}$ & $100 \mathrm{~b}$ \\
Control & $0 \mathrm{a}$ & $10 \mathrm{a}$ \\
\hline
\end{tabular}

